\title{
Practical Considerations of Applying Body Scanning as a Teaching and Research Tool
}

\author{
Simeon GILL*1, Paula WREN², Kathryn BROWNBRIDGE², Steve HAYES ${ }^{2}$, \\ Anastasiia PANCHENKO ${ }^{2}$ \\ ${ }^{1}$ The University of Manchester, Manchester, UK; \\ ${ }^{2}$ Manchester Metropolitan University, Manchester, UK
}

http://dx.doi.org/10.15221/14.259

\begin{abstract}
Used in specific ways, body scanning technology can provide greater understanding of the body and its relationship to clothing. However, body scanning is still a developing technology and little is known about the practical applications and limitations of its operation within the education environment. Further to this, whilst there have been many high profile body scanning surveys, there still appears to be little accessible information on the practical issues of body scanning or data derived from scanning. With reference to live industry and academic projects, and the application of this technology within a UK university, recommendations are provided in the following areas: supporting structures and methods to enable; long term research, short term; accruing of data that can be seen as comparable to that collected in other locations and by other teams. Importantly processes and data captured during scanner use have little standardisation and each operator is expected to develop their own structures for training, data collection, assessment, storage and application. This can be extremely difficult without accessible examples of structures and processes previously applied. The team operating this scanner have found a diverse number of uses and developed protocols for ethics and storage that ensure data collected can have long term relevance as a resource in the increasing global arena of big data and collaboration anticipated by existing funding sources. Fundamentally, recommendations are made regarding body scanner supplier interventions that would enable the easier application of this technology and ensure data is comparable and has the greatest value for each and all operators. It is hoped through the adoption and provision of resources to support consistent scanning and data storage the current data collected on a small scale by varied users can have the greatest opportunity for future use collaboratively across different user groups.
\end{abstract}

Keywords: 3D body scanning, product development, fashion education, ethics, anthropometric exploration.

\section{Introduction}

Body scanning, as a relatively new measurement technology, offers great opportunities when assessing the body for clothing product development. Historically scanning has been celebrated as a revolutionary method of collecting data of large populations used to inform sizing systems for mass produced clothing. However although many large scale surveys [1, 2] have been conducted, they have arguably not benefitted industry or improved sizing practice as much as was predicted. This paper is written from the perspective of the clothing practitioner and assesses the application of scanning technology within clothing practice. It identifies a number of areas developed to support the practice of using scanning as well as problematic areas that have hindered progress and the ability to develop effective working methods that could be used to benefit industry.

There are unique challenges to body scanner users who are trained in traditional manual methods of measurement for clothing product development and the ability to contextualise data derived from the scanner is not always easy. Further difficulties arise as unlike traditional manual measurement methods there is limited detailed information describing the undertaking of surveys, the definitions of measurement and the analysis of data. Much of the undertaking and analysis of early measurement surveys provides clear instructive guidance on all aspects of collecting and analysing the data $[3,4]$ and this has often influenced more up to date surveys and guidance on product development literature [5-7]. The information available in the public domain for major body scanning surveys is scant and very few experienced users and companies have detailed data on measurement definitions, which are central to applying the resulting scan data accurately in product development [8]. This scenario provides a set of unique problems for users of body scanning and this paper will focus on some of these issues as well as the required infrastructure to run body scanners in an 
educational environment. Whilst the teams experience of body scanning is related to the practice of operating TC2 scanners for academic and industry projects, this experience has evolved in the wider context of increasing use and possibilities of body scanning. Therefore whilst reflective experiences detailed in this paper relate directly to the TC2 scanner, the issues are more universal in nature and reflect some key considerations of body scanning for product development.

\section{Understanding body scanning}

Body scanning as the means of capturing $3 \mathrm{D}$ objects and rendering them in electronic environments through image capture devices is recognised to provide greater depths of data than manual methods of defining the body for clothing [9]. Not only does it support advanced methods of analysing the body [10-12], there is also the opportunity to explore new methods of product development like pattern development from the scanned body $[13,14]$. There are even standards, to guide on its application as a measurement tool [15], though these are merely reference measurements [16], which do not adequately recognise the possibilities of this new technology [17]. An important consideration is that these standards have evolved from manual anthropometric methods which require visual and tactile analysis of the skin to successfully locate landmarks from the skeletal structure. 3D-bodyscanning relies on surface geometry, where the body surface is formed as a mesh comprising of a collection of co-ordinate points and triangles [18] This non-contact method of measurement eliminates the possibility to use traditional palpation to identify landmarks on the body, therefore the level of precision when detecting and locating landmarks is dependent on a clearly written description which inform the programmed algorithm. Explicit information of the descriptions informing the algorithms and the algorithms themselves are commercially sensitive proprietary information and, as such, part of a company's competitive advantage, which results in a reluctance to divulge information ([19]. Consequently, without this information, it is difficult to ascertain whether the software has accurately located the landmarks without further investigation at the individual scan analysis stage. Whilst the body scanner has had extensive use in national measurement surveys [1] and has been validated as offering comparable measurements to manual methods [20], little is known about the practical applications of this technology. In contrast much of the methods and even some of the data of historic surveys are published and accessible to guide on future developments [3, 4, 21, 22]. Details from existing 3D scanning surveys can be found within disparate literature sources, though none provides the level of detail found within historic literature on measurement surveys.

Several international anthropometric surveys have been undertaken to collect population data of body size and shape. Retailers, academics and government departments have together developed surveys, such as SizeUK [23], SizeUSA [24] and Size Korea [25]. The details of each survey can be found in two ways. The first being the marketing literature which accompanies the scanner brand. A visit to TC[2] (website?) promotes its involvement in the SizeUSA survey and Human Solutions (Vitus) also publicises its involvement with Size Germany. The information provided is scant and written as an aid to market the products and services. The second is through webpages which have been developed to broadly discuss the survey and provide limited details of the outcomes. For example SizeUK, $2004 \quad$ (http://www.sizemic.eu/sizeuk.html); Size Germany, (2008) (http://www.hohenstein.de/ximages/1399929_sgabschlus.pdf); $\quad$ SizeUSA, 2004 (http://www.nytimes.com/2004/03/01/us/sizing-up-america-signs-of-expansion-from-head-to-toe.html); AIST Research Institute of Human Engineering for Quality Life - Japan, 2003 (https://www.dh.aist.go.jp/database/fbodyDB/), all provide information of sample size, demographic, gender, amount of measurements taken, brand of scanner used, funding and participating bodies and in some cases but not all general results are provided at a price. In contrast the published details within many manual surveys is considerable and includes valuable information such as: how landmarks are first defined, measurement descriptions, methods for calibrating instruments, and how the participant should be instructed. It appears to be only possible to access small 'tasters' of the 3D scanner outputs, in an aim to encourage the viewer to buy the information, but informative to support the practice of using body scanning for human measurement is very difficult to find.

Much of this technology has been developed outside of the skillsets of clothing product development practitioners and is published in areas often unrelated to product development. There are clear details of how systems of automated landmark location may work in scanning [26], however, these do not accompany the software, so a user is not able to directly understand how a landmark is identified. This reflects a similar lack of clarity in relation to the methods used to locate landmarks when manually measuring the body. [8], However clear and detailed definitions of the landmarks used for manual measurement are provided within literature. As the fundamental first step in measurement, 
landmark descriptions are crucial to understand what the measurement is and then build on how this relates to product development. The lack of transparency regarding the way 3D scanners locate body landmarks therefore prevents users from being able to fully understand the resulting measurement data, hindering the process of data application within product development.

\section{Practical application of body scanning in HE.}

The application of new technologies such as body scanning demands the acquisition of a completely new set of skills. Clothing practitioners who adopt scanning for its informative benefits in classifying the body to inform product development must expect a steep learning curve. Not only does scanning operate in a manner slightly different to manual methods, it also requires skillsets which are not traditionally learned during product development. These include a knowledge of manual measurement techniques, manipulation of $3 \mathrm{D}$ point cloud data and the operation of engineering software. In addition, the various systems display and analyse the body in a scale and manner considerably different to traditional practice. Whilst the team using the scanner have been keen to develop new skillsets, difficulties have been found when employing these technologies collaboratively with industry partners. There are also further concerns within the research environment regarding the ethical collection and storage of body scan data, which contains sensitive data related to a person's selfidentity. The following sections provide reflective details and discussion from the different stages of collection and analysis of the data to help define future considerations. The sections are aligned to the developed practices of using body scanning within $\mathrm{HE}$ over the previous decade.

\subsection{Participant data collection and ethical considerations}

Whilst body scanners allow the opportunity to capture dimensions of the body which can be analysed within software, some key data is not always embedded with the scans. The body scanner predominantly deals with capturing 3D surface data of the body and whilst the TC2 software enables other data to be collected, this is embedded with the scan and must be recorded manually. Experiences of the team suggest there is some extra data which it is essential to record alongside the body scans, only some of which it would be suitable with respect of ethical considerations to include alongside the scan. Height and weight represent two key characteristics which are required in the analysis of scan data that are not directly captured through the scan, or not with sufficient accuracy for them to be used reliably. It has been found to be considerably difficult to establish methods of data analysis without the inclusion of height and weight. The following sections describe protocols for collecting and storing the scan data with consideration of its future use and the ethical guidelines as well as responsibilities to the participant.

\subsubsection{Collection of key data to accompany scans}

During the development of methods for collecting scan data it was established that some form of database would be required to store key personal details. Initially Excel spreadsheets were used, though these were further developed using more advanced database software (Filemaker Pro), to provide a more automated tool to collect these details alongside consent. This enabled the storage of personal details from participants in a password protected environment, separate from the 3D scan data.

To enable detailed statistical analysis height and weight were manually recorded from each participant, as these are used within many established methods for dealing with anthropometric data $[4,27]$ and are essential to the creation of sizing schemes. A means to embed these with the scans is important, though these only need to be embedded with a usable scan and would be best if stored with the scan to be input at the stage of identifying a good scan.

Further details which were recorded from each participant are outlined in the following table (Table 1)

Table 1: Data required alongside scan capture

\begin{tabular}{|l|l|}
\hline Data & Reason for collection \\
\hline Height & Required for creating subgroups of the data $[28,29]$ \\
\hline Weight & Required for creating subgroups of the data [28, 29] \\
\hline Ethnicity & This will effect proportional division of the body and possibly its shape [27,30] \\
\hline Age & Required for creating subgroups of the data [28] \\
\hline Gender & Required for creating subgroups of the data \\
\hline $\begin{array}{l}\text { Geographic } \\
\text { location }\end{array}$ & $\begin{array}{l}\text { Required to fix scan populations by geographic location when analysing data } \\
\text { nationally or globally. }\end{array}$ \\
\hline
\end{tabular}


Body scanning creates a long term digital record of a person at a fixed time and in a fixed posture, it is anticipated body scans will be used to inform product development and efforts have been made to create large scale repositories of scan data (e.g. isize). It is therefore crucial that key data can be linked to these scans, though clear consideration is required as to how data is stored and linked back to the individual without possibilities of easy identification. It is important that systems are developed to facilitate this approach and would be a natural addition to scan software. The requirement to keep some personal data separate from the scans to retain anonymity and satisfy our ethical obligations to participants required we develop a separate system to collect and store personal data. Using the scanner within academic environments also necessitated that participants were identified as students or not, this removed the assumption that all scans of a certain age group were students and therefore of a certain socio-economic and educational group. This highlights that aside from purchasing scanning technology, robust methods for collecting key demographic details are required and may not always be present with the scanner.

\subsubsection{Developing protocols for consent and codifying data}

Following development of the database for collecting personal details, methods were developed to create unique codes for each scan participant in order to maintain anonymity but still preserve a clear link between the different data types (scan and personal information). To inform participants about the scan process and resulting data, a detailed video of the process, alongside an information sheet are provided to participants. Each participant is then required to sign a consent form, detailing how the data would be used assuring anonymity, in line with typical academic ethical protocols. The collection of consent provided the opportunity to generate each participant's unique code to allow the individual scans to remain anonymous, current scan systems provide means to name the scans, however there is no guidance on what format should be used. Experiences of storing and analysing scan data suggested that some key information was important within the code to enable key aspects to be identified when all that was present was a copy of the scan. A unique eight figure code was developed that included key details to enable scans to be sorted, selected and contextualized without reference to accompanying personal details. Gender was coded as M or F, experience had shown that within the scan environment not all data could be discerned visually as belonging to either gender and having to check raised a number of issues, this was especially so when participants morphology was altered through sports participation or restrictive clothing. Age was also recorded to enable grouping of data and whilst it is accepted that age would affect body size and shape, this again was not easily and accurately determinable from visual inspect of the scans. Finally ethnicity was included in the name, again this removed presumptions about appearance of different ethnicities, though it raised issues about which guidance would be used to provide the classification. The initial first level NHS classification were selected as appropriate and provided the means to identify broad ethic groupings which had been shown to have some potential effect on segment lengths [27].

\subsubsection{Assessment of scan data}

Alongside the collection of personal details, the processes of collecting scans needed clear protocols, methods for collecting height and weight were standardised in accordance with existing guidance, though it is recognised these may vary by discipline. The nature of the body scanner and the accessibility to a number of operators required strict guidance to ensure data was collected comparably between them to have least impact on the accuracy of the data when investigated as a group. This raises issues about standardisation of practice, when data may be used as part of a larger group collected using different protocols. Any standard introduced however must be sensitive to equipment, purchased separately from the scanner and the particular skillsets of the increasingly diverse operator of this technology.

\subsection{Scan data collection and analysis}

Once the process of collecting consent, participant details and coding scans is established the stage of actually collecting scan data from participants can be undertaken. This begins the process where skillsets and new methods of data interpretation is required A number of considerations are outlined here.

\subsubsection{Determination of a usable scan}

Scan manufacturers have developed some detailed and robust methods for capturing the scan, including standardisation of posture. However, during scan capture it is not unusual for difficulties to arise in determining a suitably detailed scan, without excess noise or postural issues. The first stage 
of assessing the scan is visually to check that all parts are present and that surfaces do not show unexpected deviations These are not always immediately visible, for instance forgetting to tie up long hair may not make the scan capture fail, but may result in a scan where complete data cannot be extracted. Currently there are no documented methods for assessing scans and few automated checks within the system to highlight errors of a small nature. Protocols were developed to ensure that each scan was rotated to be viewed from different angles to check the surfaces and running an automated measurement extraction helped to visual potential problems through misplaced landmarks.

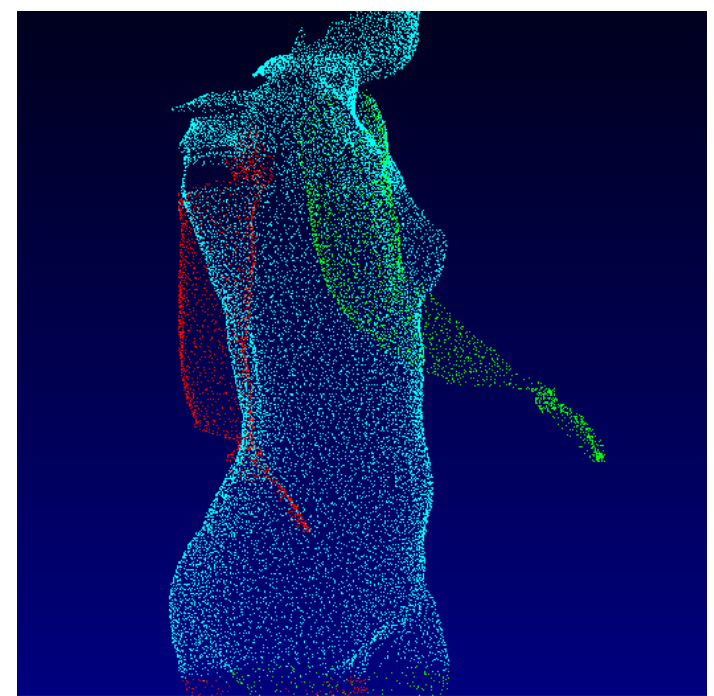

Sample of poor point cloud data on the scan

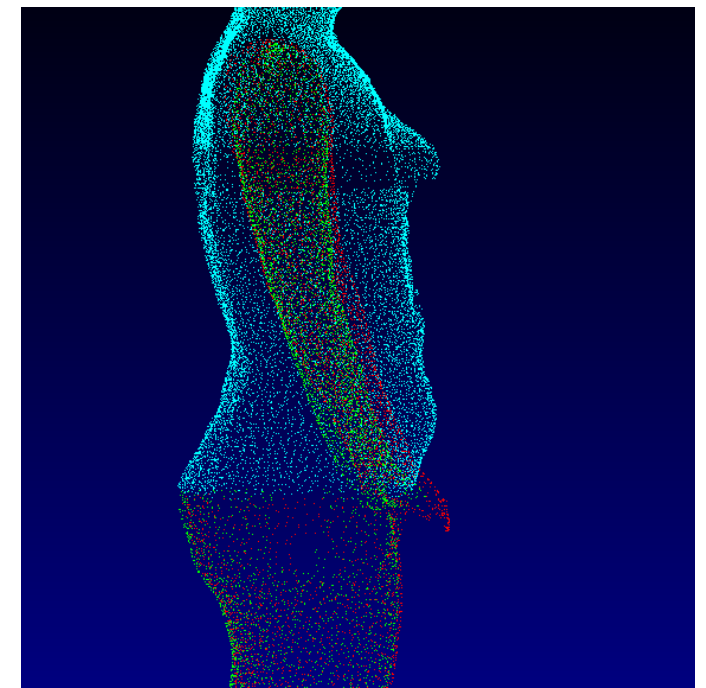

Difficulties placing crotch point and handling dark colours on the bra

Figure 1 Examples of problematic scan data

\subsubsection{Establishing which data to extract from participants}

The automated extraction facilities within the TC2 software provided a number of flexible definitions of measurements and included details of those used in national surveys. However, there were no measurements clearly aligned to existing manual standards and it became increasingly evident that body scanning using automated tools does not establish and extract measurements in a manner comparable in many cases to established manual methods. Having a list of measurements set up in accordance with existing standards $[16,31]$ would help initially integrate this technology into existing practice, even a simple translation between these and standards when directly comparable in method would help with the translation of skills. What is evident is that body scanning as indicated previously [9] provides a greater depth of data than existing manual systems and even overcomes difficulties of obtaining measurements which required a default to proportionally derived measurements within product development methods [5, 32]. Once methods of data extraction were established, the difficulty of describing the collected data, its context in terms of limited familiarity with measurement and even existing industry practices needed to be overcome. Without explicit details of landmark definitions used in body scanning it was often difficult to relate measurements directly to those with which participants or users were familiar. The mistaken notion that scanners provide comparable data to manual measurement made its application difficult in certain circumstances and highlighted the vagaries of practice existing in current methods of measurement. Further development and standardisation is required to help those experiencing scanning gain meaningful data from the process and so encourage greater uptake with the technology.

\subsubsection{Cleansing data}

Data collected from body scanning does not have the checks which occur during manual collection and to date many of the methods for error checking used in manual surveys do not currently exist within the scanning systems. This includes tolerances on measurements and the ability to check dimensions during data collection This is in part down to the sheer volume of data which can be collected. However for a number of reasons erroneous data can be collected, this may be the misplacement of landmarks due to poor point clouds, or measurement paths which are not sufficiently tied to the body surface. Issues with landmark location within the Tc2 software was found to be effected by body shape [33], though how this was dealt with, by point modification or altering 
definitions is not detailed. The lack of explicit information on how to deal with scan data removes the opportunity to develop structured and standard methods for dealing with them in future. During the process of scan cleansing it may necessary to completely delete some scans. As a consequence, new protocols were developed for capturing multiple scans and identifying those which are deemed usable. The TC2 software has a facility to rescan using the same name and appending a letter after the scan which helps in the process of identifying a good scan of an individual from a batch. However as multiple scans cannot be opened simultaneously and the best one identified, the process of establishing the best scan is not as streamlined as users would like. Often modification is required which involves manual manipulation of the landmark itself. To achieve this end clear documentation has had to be developed by the team to guide on landmark modification and ensure this process has some degree of rigour between users. However, the process of adjusting landmarks requires a familiarity with anatomy and manual measurement techniques as well as product development to ensure decisions on the placement of landmarks is suitably informed. The difficulty of determining how the landmarks are defined within the software makes it necessary to reflect manual definitions and it must then be hoped methods of defining these virtual landmarks are continuing to replicate manual methods. A common issue is found with the placement of the front underarm points, this necessitated that a definition be created which should describe the placement of this point. In manual measurement various tools have been used to define this landmark $[6,8]$ which equates to the crease formed in the axilla when the arm is abducted to the body and the skin folds just above the musculature controlling adduction. However most methods of manual landmark location are developed as a practitioner skill, with few examples of detailed descriptions of landmarks and their methods of location [8].

When adjusting the underarm landmark which is visually assessed to be misplaced, a judgement must be made as to where the landmark would lie if it were manually positioned on the body; this is complicated as the scan position requires the arm be abducted from the body. An example of the process can be seen in Figure 2. The judgement required to reposition this landmark must be made without all the visual and tactile cues of manual methods and in a scale much smaller than lifesize, as well as scant detail from the software as to what this landmark was originally positioned with reference to.

Armpit points (back and front) are located on the surface of the torso, near the crotch of arm and body. Highlighting the armscye measurement (W18) may be helpful. Manually these will be associated with the armpit folds, when the arm is adducted to the side.

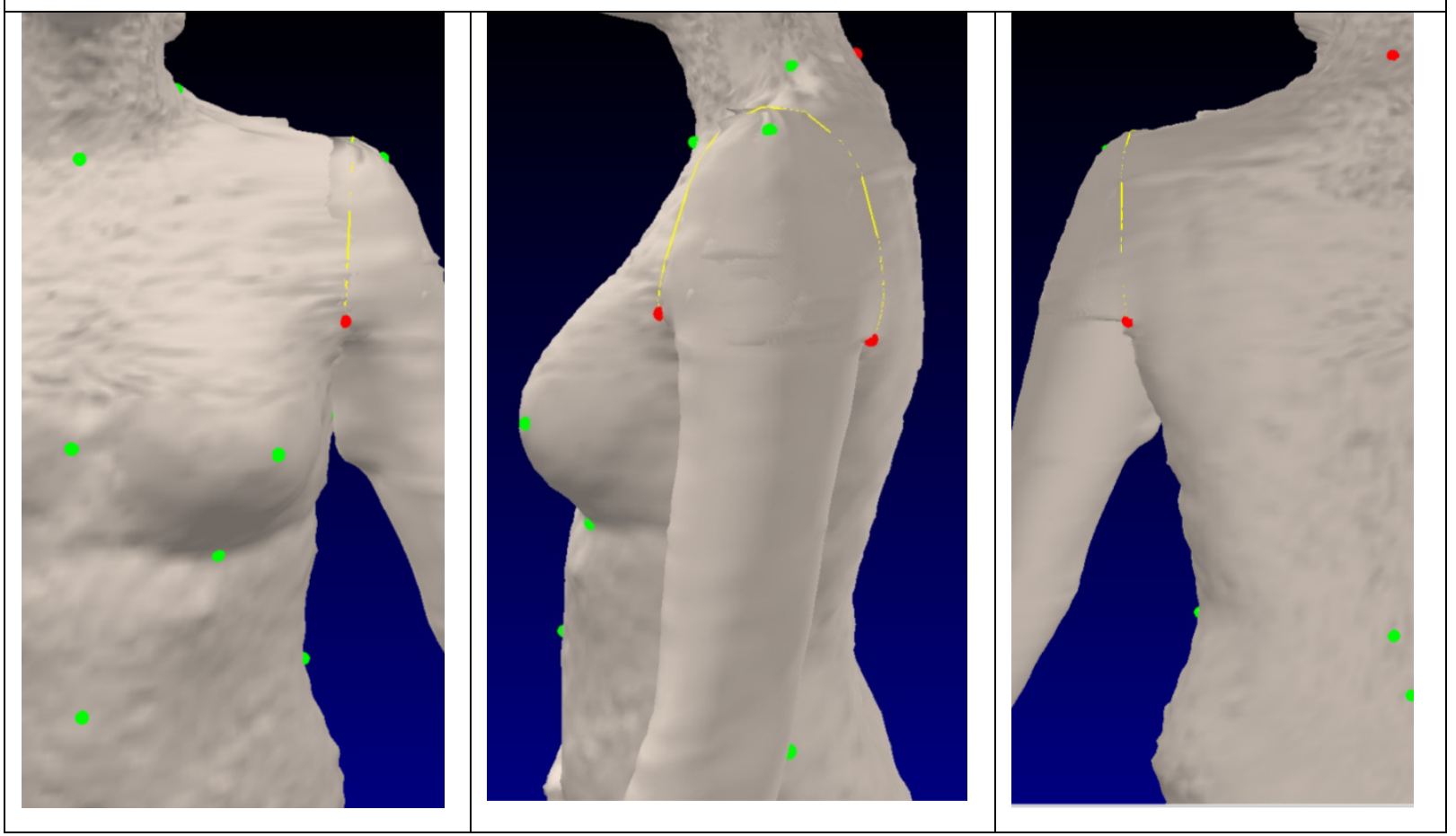

Figure 2: Visual guidance provided on correct location of the armpit point 
The necessity to create visual guidance on what is considered good landmark placement to guide new users, highlights an area of development that is key to the success of using automated measurement within body scanning with varied user groups. If persistent errors cannot be checked and amended against some standards there is the possibility of lots of erroneous data being generated by the multiple users of this technology.

\subsection{Research with body scan data}

Having access to body scanning technology has provided opportunities to create new research pathways, both concerned with understanding the data created, as well as providing new data to help understand the body within product development. The scanner has also been found to be a useful collaborative tools providing opportunities for engineers, psychologists, nutritionists and product developers to explore the body and its relationships. However, this also necessitates that there is a full understanding of the equipment and a means to explain its methods of establishing measurements and the meaning of the data presented within the software.

\subsubsection{What to give participants and how}

The body scanner provides a unique output for many participants, on screen they can view their bodies in 3D from a number of angles and printed 2D outputs provide a detailed snapshot of the body usually representing a front and side view. This visual data can be extremely informative, but often has little cultural context, due to the limited use of scanning for the general population. Further difficulties are faced when providing measurement data to participants or industry partners, due to the differences between methods of scanning and manual measurements, the data is not directly comparable. It is therefore difficult to provide an output with a clear definition that aligns to most peoples' historic experience. However explaining to participants the nuances of this becomes prohibitive, they want data which will help them make or select product and they often will not want to contend with the idea of their being more than one definition for a measurement even though it is named the same Further changes in product development practice which see industry professionals using garment measurements with no direct relation to body dimensions [34] makes the situating of scan data in their practice difficult. In certain cases the team had developed outputs which signified measurements that could be used in sizing selection, though often participants were as interested in the seeming variance between left and right sides as they were with the product related data. Without standardisation of practice and a common set of definitions between scanner systems it is difficult to see how this will be avoided in future, it is clear however that existing standards for body scanning measurement [16] are limited in their ability to address these issues, which have a direct bearing on body scanning as a usable tool that participants will engage with during product development. It is not unusual for comments to be made about comparison of manual and scanner measurements; however, this often fails to recognise the advantages of scanning and limitations of manual methods. Manual methods for product development require many dimensions be determined proportionally due to their difficulty of capture with the tools available. Body scanning in contrast provides the opportunity to capture dimensions which have much greater context in developing products and can overcome many limitations of manual methods

\subsubsection{Measurement networks}

Body scanning by its very nature requires that many measurements are related during their definition, this creates a clear measurement network, which better relates to product development than existing manual approaches (Figure 3). Products which are worn are usually formed of complete surfaces which cover areas of the body, these relate circumferences by surface lengths and can be more readily related to a measurement network, than manual methods which often deal with measurement in isolation. 


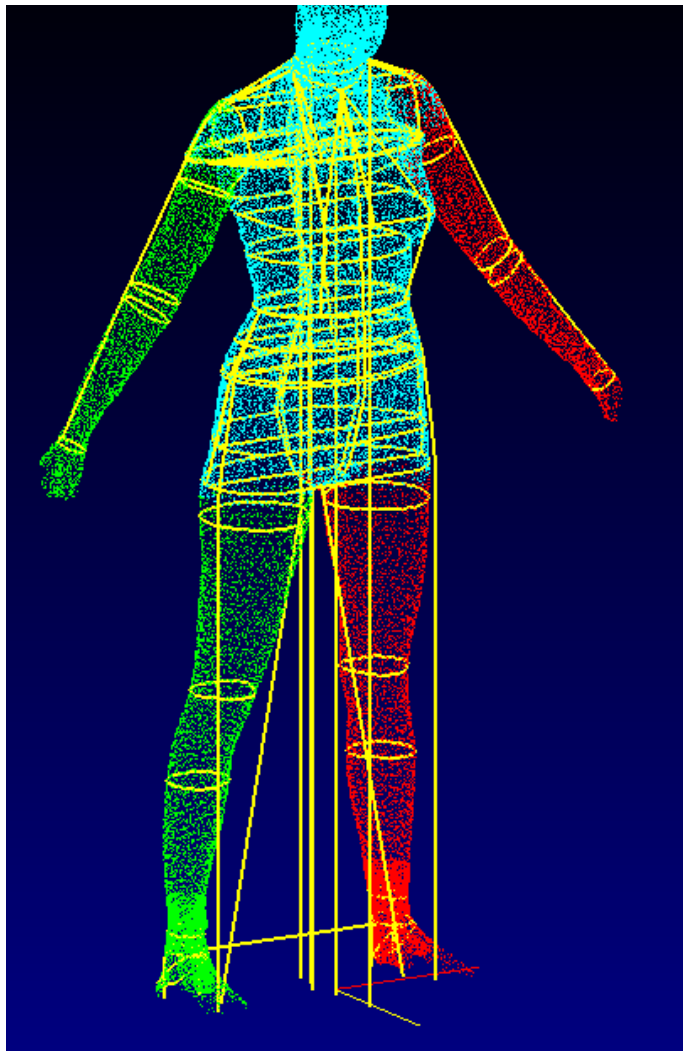

Picture of measurement network on scan

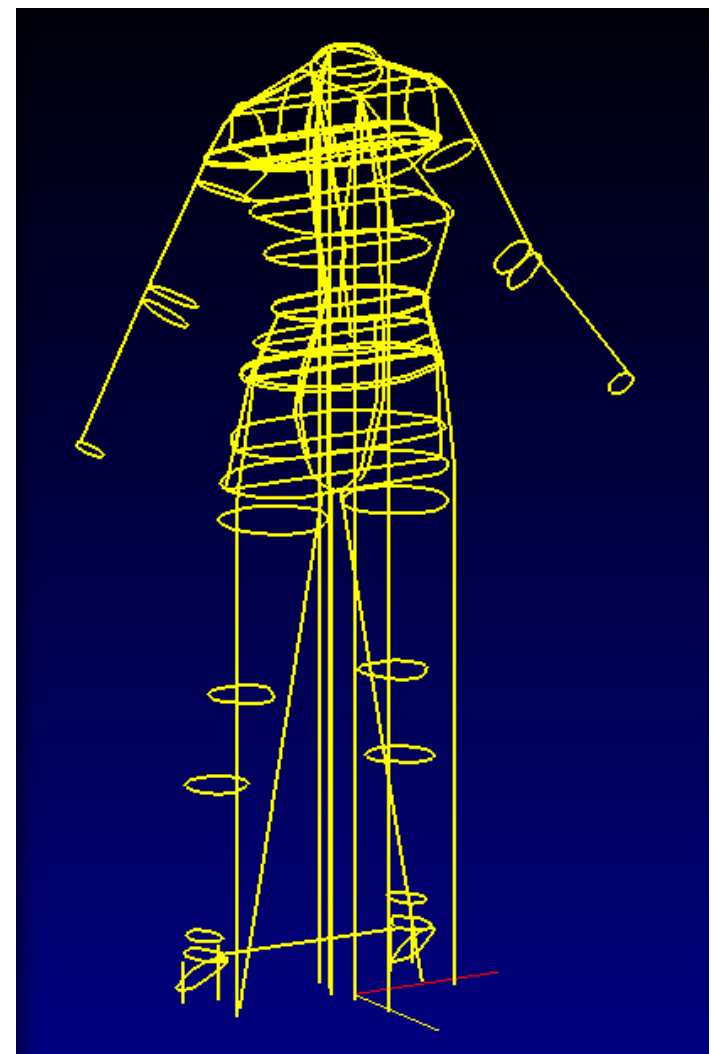

Picture of measurement network on its own

Figure 3: Measurement networks

\section{Conclusions and future considerations of body scanning for product development}

Whilst there is a clear need to for greater interventions and innovations to enable body scanning to be accessible to more practitioners and those in HE environments, it does provide some clear solutions to limited manual practices. There is a need to develop measurement definitions built on the principals of measurement networks and with sufficient levels of information for data to be understood as with historic manual surveys. The following considerations are important to establish prior to implementations body scanning in an HE environment and may have application to users outside of this. 1. Methods need to be developed for collecting demographic details to contextualise the scan data, these include at a minimum height, weight, age and ethnicity; 2. Methods of codifying data are required to protect anonymity but allow informed storage and management of data; 3 . Clear understanding of automated methods are required and clear definitions of measurements should be documented and where possible include details of virtual landmarks. 4. Robust methods must be developed for the analysis of data for accuracy and clear procedures for amending scans. 5. Training should be sought in software allowing analysis and processing of the scan data outside of the accompanying software packages.

Fundamentally this technology will be more successful if there is clear and open discussion between developers and users to ensure it can fulfil its potential and all stakeholders (suppliers, users and participants) can all benefit. Some further discussion of the considerations highlighted in the paper are in the sections below.

\subsection{Standardisation of data collection}

It is important that scan manufacturers give further consideration to the key characteristics/demographic details that are important to capture to enable scan data to have the widest application. Further to this means of coding data need to be considered and some efforts made to ensure that all users can collect data which is meaningful yet retains anonymity of the participant. 


\subsection{Development of new skillsets}

The knowledge base indicates research has developed and explored a variety of algorithmic methods enabling the software programmes within bodyscanning systems to locate and/or place landmarks over the virtual skin surface. The research is from a wide variety of disciplines including anthropometrics [35]); clothing development; computer science [18, 36] and statistics, indicating skillssets from interdisciplinary fields will be required to work together to effectively develop the technology further. This expansion of skillsets required to successfully operate body scanners suggests those being taught product development need to gain further familiarity with technical engineering software principals and techniques to fully benefit from body scanning technology. This requires that discipline specific practices are shared and made more accessible, so computer programmers can better understand product development practices and those involved in product development can better engage with methods of automated analysis of the body in electronic environments.

\subsection{Enhanced tools for analysis}

Whist existing systems of scan analysis provide good opportunities to investigate data for product development, detailed analysis often requires the use of engineering software outside of the skillsets of many product developers. Developments in visualisation within the accompanying software tools would help both the exploration of data for research, as well as the ability to communicate data to participants. Some considerations here are, the ability to open multiple scans, align them and visually compare them, this would help show participants how they compare to each other as well as to a standards, like a scanned dress form, further means to show cross sections and planes through the body at different locations would enhance how a participants can engage with the data.

\section{References}

[1] W. Yu, "Human anthropometrics and sizing systems," in Clothing appearance and fit: Science and technology, J. Fan, W. Yu, and L. Hunter, Eds., ed Cambridge: Woodhead, 2004, pp. 169-195.

[2] S. Ashdown, Ed., Sizing in Clothing, Developing effective sizing systems for ready-to-wear clothing. Cambridge: Woodhead Publishing, 2007, p.^pp. Pages.

[3] R. O'Brien and W. C. Shelton, "Womens Measurements for garment and pattern construction (Public. No. 454, Department of Agriculture)," United States Department of Agriculture1941.

[4] W. F. F. Kemsley, Ed., Women's measurements and sizes. London: Joint Clothing Council Ltd, H.M.S.O, 1957, p.^pp. Pages.

[5] P. Kunick, Modern Sizing and Pattern making for womens, mens and childrens garments. London: Philip Kunick, 1984.

[6] A. Beazley, "Size and fit: Procedures in undertaking a survey of body measurements - Part 1," Journal of Fashion Marketing and Management, vol. 2, pp. 55-85, 1997.

[7] A. Beazley and T. Bond, Computer-aided pattern design and product development. Oxford: Blackwell Science, 2003.

[8] S. Gill, "Determination of Functional Ease Allowances using anthropometric measurement for application in pattern construction," unpublished PhD Thesis, Manchester Metropolitan University, 2009.

[9] E. Bye, K. L. LaBat, and M. R. Delong, "Analysis of Body Measurement Systems for Apparel," Clothing and Textiles Research Journal, vol. 24, pp. 66-79, 2006.

[10] J. Y. Lee, C. L. Istook, Y. J. Nam, and S. M. Park, "Comparison of body shape between USA and Korean women," International Journal of Clothing Science and Technology, vol. 19, pp. 374-391, 2007.

[11] S. Ashdown, S. Choi, and E. Milke, "Automated side-seam placment from 3D body scan data," International Journal of Clothing Science and Technology, vol. 20, pp. 199-213, 2008.

[12] K. Brownbridge, S. Gill, and S. Ashdown, "Effectiveness of 3D Scanning in Establishing Sideseam Placement for Pattern Design," in 4th International Conference on 3D Body Scanning Technologies, Long Beach California, USA, 2013.

[13] A. Sayem, S,M., R. Kennon, and N. Clarke, "Resizable trouser template for virtual design and pattern flattening," International Journal of Fashion Design, Technology and Education, vol. 5, pp. 55-65, 2012.

[14] X. Tao and P. Bruniaux, "toward advanced three-dimensional modeling of garment prototype from draping technique," International Journal of Clothing Science and Technology, vol. 25, pp. 266-283, 2013. 
[15] BSI, "BS EN ISO 20685:2010: 3-D scanning methodologies for internationally compatible anthropometric databases," ed. London: British Standards Institute, 2010.

[16] ISO, "7250-1:2010: Basic human body measurements for technological design - Part 1: Body measurement definitions and landmarks," ed. Brussels: International Standards Organisation, 2010.

[17] D. Tyler, A. Mitchell, and S. Gill, "Recent advances in garment manufacturing technology; joining techniques, 3D body scanning and garment design," in The global textile and clothing industry, R. Shishoo, Ed., ed Cambridge, UK: Woodhead Publishing, 2012, pp. 131-170.

[18] R. Suikerbuik, H. Tangelder, H. Daanen, and A. Oudenhuijzen. (2004, 15 January 2010). Automatic Feature Detection in 3D Human Body Scans. Available: http://www.cs.uu.nl/groups/AA/multimedia/publications/pdf/suikerbuik04.pdf

[19] K. P. Simmons and C. L. Istook, "Body measurement techniques: Comparing 3D bodyscanning and anthropometric methods for apparel applications," Journal of Fashion Marketing and Management, vol. 7, pp. 306-332, 2003.

[20] J. P. Bougourd, L. Dekker, P. Grant Ross, and J. P. Ward, "A Compilation of Women's Sizing by 3D Electronic Scanning and Traditional Anthropometry," Journal of The Textile Institute, vol. 91, pp. 163-173, 2000.

[21] C. E. Clauser, I. O. Tebbetts, B. Bradtmiller, J. T. McConville, and C. C. Gordon, "Measurers Handbook: US Army Anthropometric Survey," US Army Natick Research Development \& Engineering Centre, Natick, MA TR-88/043, 1988.

[22] J. Roebuck, Anthropometric Methods: Designing to fit the human body. Santa Monica, CA: Human Factors and Ergonomics Society, 1995.

[23] J. C. K. Wells, P. Treleaven, and T. J. Cole, "BMI compared with 3-dimensional body shape: the UK National Sizing Survey," American Journal of Clinical Nutrition, vol. 85, pp. 419-425, 2007.

[24] S. Loker, S. P. Ashdown, L. Cowie, and S. K. A. (2004, 19/09/2006). Consumer interest in commercial applications of body scan data. Journal of Textile and Apparel, Technology and Management. Journal of Textile and Apparel, Technology and Management 4/1(4/1). Available: www.tx.ncsu.edu/jtatm/volume4issue1/articles/Loker/Loker full 100 04.pdf

[25] H. Han, Y. Nam, and K. Choi, "Comparative analysis of 3D body scan measurements and manual measurements of size Korea adult females," Journal of Industrial Ergonomics, vol. 40, pp. 530-540, 2010.

[26] H. Han, Y. Nam, and S. H. Shim, "Algorithms of the Automatic Landmark Identification for various torso shapes," International Journal of Clothing Science and Technology, vol. 22, pp. 343-357, 2010.

[27] J. Winks, Clothing Sizes international standardisation. Manchester, UK: The Textile Institute, 1997.

[28] J. Croney, Anthropometry for designers. London: Batsford, 1980.

[29] A. Beazley, "Size and fit: The development of size charts for clothing - Part 3," Journal of Fashion Marketing and Management, vol. 3, pp. 66 - 84, 1999.

[30] A. Mastamet-Mason, H. M. De Klerk, and S. Ashdown, "Identification of a unique African female body shape," International Journal of Fashion Design, Technology and Education, vol. 5, pp. 105-116, 2012.

[31] ISO, 8559:1989 Garment construction and anthropometric surveys - Body dimensions: International Standards Organisation, 1989.

[32] W. H. Hulme, The theory of garment-pattern making, 2nd ed. London: The National Trade Press, 1946.

[33] P. Devarajan and C. L. Istook, "Validation of 'Female Figure Identification Technique (FFIT) for Apparel' Software," Journal of Textile and Apparel, Technology and Management, vol. 4, pp. 1-22, 2004.

[34] P. Wren, "The garment fitting process: the inter-related issues and problems that impact upon clothing fit and how industry personnel and procedures address poor fit," Unpublished MSc Thesis, Manchester Metropolitan University, Manchester, 2009.

[35] L. McKinnon and C. L. Istook, "Body scanning: The effects of subject respiration and foot positioning on the data integrity of scanned measurements," Journal of Fashion Marketing Management, vol. 6, pp. 103-121, 2002.

[36] W.-C. Chao and E. Wang, Min-yang, "An approach to estimate body dimensions through constant body ration benchmarks," Applied Ergonomics, vol. 42, pp. 122-130, 2010. 